\title{
BM] Global Health BCG vaccination is associated with reduced malaria prevalence in children under the age of 5 years in sub- Saharan Africa
}

\author{
Mike LT Berendsen (D) , , ${ }^{1,3,4}$ Sjors WL van Gijzel, ${ }^{1}$ Jeroen Smits, ${ }^{5}$ Quirijn de Mast, \\ Peter Aaby, ${ }^{4}$ Christine S Benn, ${ }^{2,3}$ Mihai G Netea, ${ }^{1,6,7}$ Andre JAM van der Ven ${ }^{1}$
}

To cite: Berendsen MLT, van Gijzel SWL, Smits J, et al. BCG vaccination is associated with reduced malaria prevalence in children under the age of 5 years in sub-Saharan Africa. BMJ Global Health 2019;4:e001862. doi:10.1136/ bmjgh-2019-001862

\section{Handling editor Sanni Yaya}

- Additional material is published online only. To view, please visit the journal online (http://dx.doi.org/10.1136/ bmjgh-2019-001862).

Received 22 July 2019 Revised 26 September 2019 Accepted 19 0ctober 2019
Check for updates

C Author(s) (or their employer(s)) 2019. Re-use permitted under CC BY-NC. No commercial re-use. See rights and permissions. Published by BMJ.

For numbered affiliations see end of article.

\section{Correspondence to} Mike LT Berendsen; Mike.Berendsen@radboudumc. $\mathrm{nl}$

\section{ABSTRACT}

Introduction Malaria continues to be a major cause of morbidity and mortality in sub-Saharan Africa (SSA) without effective interventions. Bacillus Calmette-Guérin (BCG) vaccine possesses protective non-specific effects, which extend beyond protection against tuberculosis. This study explores whether BCG is associated with protection against malaria in children under the age of 5 years in SSA. Methods We used data from the Demographic Health Survey programme, including 34206 children from 13 SSA countries. BCG status was taken from vaccination cards when present; if not, mother's recall was used. Presence of malaria was defined as a positive rapid diagnostic test. Maternally reported presence or absence of fever in the previous 2 weeks defined symptomatic status. Multilevel logistic regression was used to account for the two-stage cluster sampling method.

Results Of the 34206 children, 12325 (36.0\%) children were malaria positive and $29766(87.0 \%)$ were BCG vaccinated. After correction for relevant child, maternal and household factors, BCG vaccination was associated with a lower malaria prevalence (adjusted $\mathrm{OR}(\mathrm{aOR})=0.94$, $95 \% \mathrm{Cl} 0.90$ to 0.98 ), especially among children of whom BCG information was retrieved from a vaccination card $\left(\mathrm{aOR} \mathrm{R}_{\text {card }}=0.88,95 \% \mathrm{Cl} 0.82\right.$ to 0.94$)$. Restricting the analysis to children from regions with suboptimal BCG coverage increased the association $\left(\mathrm{aR}_{\text {card }}=0.81,95 \% \mathrm{Cl} 0.73\right.$ to 0.89 ). We observed an increasingly beneficial association with each month of age of the child $\left(\mathrm{aOR}_{\text {card }}=0.996,95 \%\right.$ $\mathrm{Cl} 0.993$ to 0.999). BCG associations were similar for asymptomatic ( $\mathrm{aOR}_{\text {card }}=0.86,95 \% \mathrm{Cl} 0.81$ to 0.92$)$ and symptomatic ( $\mathrm{aOR}_{\text {card }}=0.89,95 \% \mathrm{Cl} 0.78$ to 1.01$)$ malaria. Conclusions BCG vaccination is associated with protection against malaria. This protection is highest in regions with suboptimal BCG coverage. These results indicate a possible role for timely BCG vaccination in the protection of malaria and its elimination by reducing the transmission reservoir. If confirmed in further research, our findings have substantial implications for global efforts to reduce malaria burden.

\section{INTRODUCTION}

Malaria continues to be a major threat, despite advances under the United Nations Millennium Development Goals (MDGs). ${ }^{1}$

\section{Key questions}

What is already known?

- Bacillus Calmette-Guérin (BCG) vaccine has beneficial effects on child mortality and morbidity that cannot be attributed to protection against tuberculosis.

- In studies on murine malaria and in a controlled human malaria model, BCG vaccination increased immune responses, which were associated with protection.

- Whether BCG vaccination is able to decrease malaria prevalence at a population level in humans remains unclear, since the three epidemiological studies that have been performed were heterologous in design and outcome and had limited generalisability.

What are the new findings?

- Our large, multinational study suggests that BCG vaccination is associated with a reduced risk of malaria in children under the age of 5 years in subSaharan Africa.

- The association between BCG vaccination and malaria was largest in regions with suboptimal BCG coverage.

What do the new findings imply?

- If confirmed, our findings imply that timely BCG vaccination could aid the global efforts to reduce malaria burden.

- Substantial gains can be reached with this established and cheap vaccine, especially in countries with suboptimal BCG coverage, countries that are suffering most from the global malaria burden.

After years of decline, no significant progress in reducing global malaria burden was made during 2015-2017. Numbers are still staggering, with 3.1 billion people at risk of an infection, as well as 219 million malaria cases and 435000 deaths worldwide. ${ }^{2}$ These numbers highlight the persisting problem of malaria that affects mainly children under the age of 5 years in sub-Saharan Africa 
(SSA), and they press for the need for effective interventions.

The design of malaria vaccines is being extensively investigated on the premise that vaccines have significantly decreased the mortality and morbidity of other major infectious diseases. Moreover, general distribution of malaria vaccines could add to control of the infectious reservoir of asymptomatic carriers. ${ }^{3}$ However, the multistage development of the parasite complicates vaccine design, ${ }^{4}$ and although substantial progress has been made in recent years, routine malaria vaccination seems for now to be restricted to pilot studies. ${ }^{5}$ Alternative options to increase protection against malaria might be found in currently used routine vaccines. Numerous studies have shown that vaccines have additional effects to the initially intended protection against their target diseases, the so-called 'non-specific effects (NSEs) '. ${ }^{6-9}$ Beneficial NSEs seem to be restricted to live-attenuated vaccines, such as bacillus Calmette-Guérin (BCG) ${ }^{10}$ Evidence has suggested that BCG vaccination reduces overall mortality and morbidity more than would be expected from its 'specific' effect on tuberculosis, for instance, by reducing the risk of sepsis and acute lower respiratory tract infections. ${ }^{711}$ These non-specific BCG effects seem to be influenced by timing of vaccination. ${ }^{9} 1012$

This raises the question whether these NSEs of BCG can also offer protection against malaria infection. A role for BCG in protection against murine malaria infection was already demonstrated in 1976, where BCG vaccination of mice reduced levels of parasitaemia and increased survival. ${ }^{13}$ In contrast, little is known about effects in humans. Recently, in a controlled human malaria infection model, we have shown that BCG vaccination lowered parasitaemia levels in a subgroup of vaccinees. ${ }^{14}$ Whether BCG vaccination is able to decrease malaria prevalence at a population level remains unclear. The few epidemiological studies that have been performed are heterologous in design and outcome and have limited generalisability. ${ }^{15-17}$ To examine a possible protective role of BCG against malaria in humans, this study focusses on the NSEs of routine BCG vaccination on malaria prevalence in children under the age of 5 years in SSA. In addition, we explored the association with malaria prevalence by timing of vaccination and by symptomatic status.

\section{METHODS \\ Datasets}

This study used multiple datasets with retrospective and cross-sectional data from the Demographic Health Survey (DHS) Programme. The DHS Programme collects data on fertility, family planning, HIV/AIDS, maternal and child health, vaccinations and nutrition from over 90 lowincome countries, using continuously updated surveys. ${ }^{18}$ Selected surveys include information on malaria diagnosis, and these were derived from the Database Developing World (www.globaldatalab.org). These surveys were Angola 2016, Benin 2012, Burkina Faso 2010, Cameroon
2011, Democratic Republic of Congo 2014, Ivory Coast 2012, Gambia 2013, Ghana 2014, Guinea 2012, Mali 2013, Mozambique 2011, Rwanda 2015, Senegal 2011, Tanzania 2016 and Togo 2014. The combined dataset was recoded to make sure each individual child was a separate case, resulting in a dataset with 541061 children.

\section{Study population}

All children aged 0-60 months who had a malaria rapid diagnostic test (RDT) result and from whom valid BCG information was available were included. Children with missing values for day of birth had the middle day of the month imputed as their day of birth (15 for February and 16 for all other months). When they had received BCG and/or oral polio 0 vaccine in their month of birth, the middle day between start of the month and the day of vaccination was used. After exclusion of children over the age of 5 years (384672) or without information on malaria diagnosis (90429) or BCG vaccination (5909), 60051 children remained in the dataset.

All regions with less than $10 \%$ malaria were excluded to ensure convergence of a multilevel model and to prevent inclusion of regions with sporadic malaria infections, resulting in a small and specific malaria infected subgroup. This resulted in exclusion of 55 out of 170 regions: 1 region from Democratic Republic of Congo, Ghana, Guinea, Mali and Togo; 2 regions from Benin and Mozambique; 3 regions from Rwanda; 8 regions from Angola; 13 regions from Tanzania; and all the regions from Senegal (14) and The Gambia (8). After this exclusion, the dataset was finalised with 43040 children, which represented 34206 children after inclusion of the DHS weights.

\section{Core variables and covariates}

Data collection was performed according to DHS protocol. Information on BCG was retrieved from vaccination cards on visit for $17440 / 34206$ children (51.0\%). If these cards were not present, the information as reported by the mother was used $(12325 / 34206(36.0 \%))$. The remaining 4441/34206 children $(13.0 \%)$ had not undergone BCG vaccination. Associations with the timing of BCG vaccination were studied with a continuous variable, as well as a categorical variable with the categories: 'not vaccinated' (reference), 'at date of birth', 'between 1 day and 1 week', 'between 1 week and 1 month', 'after 1 month' and 'timing unknown'.

Malaria status was determined in a representative subsample of all children under the age of 60 months using an RDT on capillary blood samples obtained through a finger or heel prick at the time of conducting the survey. Consent was asked after reading a consent statement to the parent or responsible adult. Throughout the countries assessed, approved RDTs from different manufacturers and with different specificity were used (online supplementary table 1). In addition, 9 out of the 13 countries (excluding Angola, Cameroon, Ghana and Guinea) performed microscopy of thick-smear slides. 
Symptomatic malaria was defined as having a positive RDT and a history of fever in the previous 2 weeks based on mothers' recall. A positive RDT with the absence of fever was defined as asymptomatic malaria.

The association between BCG vaccination and malaria was corrected for covariates at the level of the child, the mother and the household. Inclusion of covariates was based on literature, expert opinion and availability in DHS data. An age-independent variable was created for vaccinations, which indicated whether the child had received all vaccines (excluding BCG) for his/her age, according to the countries' vaccination schedule at the time of malaria testing. Household wealth was measured by the International Wealth Index. ${ }^{19}$ To address for variables with missing data, the dummy variable adjustment procedure was used. ${ }^{20}$

\section{Statistical analyses}

DHS datasets are hierarchical, meaning that households are nested in sample clusters, which are nested in regions, which are nested in countries. To address this nesting, the association between BCG vaccination and malaria prevalence was studied with a multilevel logistic regression model including four levels: country, region, cluster and child. To account for the two-stage cluster sampling method at which the data were primarily sampled, modified weights assigned by the DHS were used to obtain representative samples. First, DHS weights were denormalised using the UN World Population Prospects to allow for analysis of pooled data. ${ }^{21}$ Second, as multilevel analysis requires weights at each stage of sampling, weights were split into cluster weights and individual weights. Information to generate the cluster weights is not provided in DHS data. Therefore, estimates of the cluster weights were based on the number of households for an average cluster within a region per country using appendix A of the DHS final reports. For Democratic Republic of Congo and Mozambique, appendix A did not state the number of households per cluster and the average numbers of all other countries combined was used. The individual weight was thereafter calculated by dividing the denormalised overall DHS weight by the cluster weight. Finally, sample weights were adjusted for the number of children per mother so that their application did not increase sample size.

Robustness of the association was checked by exclusion of regions with over $90 \%$ BCG vaccination coverage (avoidance of a small and specific subgroup of unvaccinated children), exclusion of children under the age of 6 months (likely to have protective maternal antimalarial antibodies) and inclusion of thick smear-based malaria diagnosis.

The independence of the association between BCG and malaria prevalence was tested by inclusion of interactions with sex and age (as a proxy for time since vaccination). A squared variable of age was included to account for non-linear correlations. Differences in the association for asymptomatic and symptomatic malaria were analysed with separate multilevel logistic regressions.

Data analyses were done using SPSS V.24, STATA V.14 and MLWin V.2.35. Multilevel regression models were run in MLWin using the runmlwin command from STATA. A $p$ value of $<0.05$ was considered statistically significant.

\section{Patient and public involvement}

There has been no patient and/or public involvement in the study design, data collection, data analysis and writing of this research.

\section{RESULTS}

This study examined the association between BCG vaccination and malaria prevalence in 34206 children under the age of 5 years from 13 countries in SSA. Characteristics of the population are displayed in table 1. Malaria RDT was positive in $36.0 \%$ (12325/34206) of the children and varied widely between countries, with only $13.2 \%$ (89/675) in Rwanda up to $75.4 \%$ (1699/2254) in Burkina Faso. Overall, BCG coverage was $87.0 \%$ (29765/34206) with less extreme, but clearly noticeable intercountry variation ranging from $60.7 \%(638 / 1051)$ in Angola to 99.3\% (670/675) in Rwanda (figure 1A and online supplementary table 2). Apart from country differences, we noted clear regional differences (figure 1B). Children who had undergone BCG vaccination were of the same age and sex as unvaccinated children, but had on average better health and housing conditions than their unvaccinated counterparts.

The group of children who were BCG vaccinated had a lower malaria prevalence than children who were not vaccinated $(\mathrm{OR}=0.79,95 \%$ CI 0.75 to 0.83$)$. This difference remained after correction for covariates at the level of the child, mother and household ( $\mathrm{aOR}=0.94,95 \%$ CI 0.90 to 0.98$)$ ) (figure 2A). Only children of whom BCG information was retrieved from a vaccination card had lower odds on malaria $\left(\mathrm{aOR}_{\text {card }}=0.88,95 \%\right.$ CI 0.82 to 0.94 ), while children in whom BCG vaccination was recalled by their mother did not $\left(\mathrm{aOR}_{\text {recall }}=0.97,95 \%\right.$ CI 0.91 to 1.02), owing to possible misclassification in the latter (figure 2A and table 2).

\section{Thick smears, BCG coverage and maternal antimalarial antibodies}

Microscopy of thick smears is still the golden standard of malaria diagnosis in many endemic countries even though RDTs often show similar diagnostic accuracy. ${ }^{22}$ Nevertheless, malaria prevalence could have been underestimated due to species-specific RDTs and poorer performance at lower parasite densities. Among the children with both RDT and thick-smear results, 5.3\% (1373/26131) was RDT negative and thick-smear positive (online supplementary table 3). Changing their malaria status to positive only marginally influenced the association between BCG and malaria ( $\mathrm{aOR}_{\text {card }}=0.86,95 \%$ CI 0.79 to 0.92 ); $\mathrm{aOR}_{\text {recall }}=0.94,95 \% \mathrm{CI} 0.88$ to 1.00 ) (figure $2 \mathrm{~B}$ and online supplementary figure 1). There was also a substantial 


\section{BMJ Global Health}

Table 1 Population characteristics of all 34206 children, overall and separated by BCG vaccination status

\begin{tabular}{|c|c|c|c|c|}
\hline Item & $\begin{array}{l}\text { All children } \\
\mathrm{N}=34206\end{array}$ & $\begin{array}{l}\text { Not } B C G \text { vaccinated } \\
n=4441\end{array}$ & $\begin{array}{l}\text { BCG vaccinated } \\
\mathrm{n}=29765\end{array}$ & P value* \\
\hline \multicolumn{5}{|l|}{ Child level } \\
\hline Malaria RDT & & & & $<0.0001$ \\
\hline Negative & $21880(64.0)$ & $2380(53.6)$ & $19500(65.5)$ & \\
\hline Positive & 12325 (36.0) & $2061(46.4)$ & $10265(34.5)$ & \\
\hline Malaria blood slide $\dagger$ & & & & $<0.0001$ \\
\hline Negative & 18925 (72.4) & $2117(63.2)$ & $16809(73.8)$ & \\
\hline Positive & $7206(27.6)$ & $1234(36.8)$ & $5972(26.2)$ & \\
\hline Age (months), median (IQR) & $27.47(23.66)$ & 28.25 (24.89) & $27.37(23.43)$ & 0.11 \\
\hline Birth order number, median (IQR) & $3(3)$ & $3(4)$ & $3(3)$ & $<0.0001$ \\
\hline Sex & & & & 0.59 \\
\hline Male & $17330(50.7)$ & $2267(51.0)$ & $15064(50.6)$ & \\
\hline Female & $16875(49.3)$ & $2174(49.0)$ & $14701(49.4)$ & \\
\hline Twin & & & & 0.015 \\
\hline No & $33448(97.8)$ & $4364(98.3)$ & $29084(97.7)$ & \\
\hline Yes & $758(2.2)$ & $76(1.7)$ & $681(2.3)$ & \\
\hline Place of delivery $\dagger$ & & & & $<0.0001$ \\
\hline Non-hospital & 12148 (35.6) & $3085(70.3)$ & $9063(30.5)$ & \\
\hline Public & 18868 (55.3) & $1130(25.8)$ & $17738(59.7)$ & \\
\hline Private & $3073(9.0)$ & $173(3.9)$ & $2900(9.8)$ & \\
\hline Size at birth† & & & & $<0.0001$ \\
\hline Small & 4141 (12.4) & $653(15.6)$ & 3488 (11.9) & \\
\hline Average & $15158(45.3)$ & $1862(44.5)$ & $13297(45.4)$ & \\
\hline Large & $14198(42.4)$ & $1668(39.9)$ & $12530(42.7)$ & \\
\hline Preceding birth interval,$\uparrow$ median (IQR) & $35(23)$ & $32(18)$ & $35(24)$ & $<0.0001$ \\
\hline Weight z-score, † mean (SD) & $-1.02(1.26)$ & $-1.29(1.39)$ & $-0.98(1.23)$ & $<0.0001$ \\
\hline Breast feeding $\dagger$ & & & & $<0.0001$ \\
\hline Never & $742(2.2)$ & $144(3.3)$ & $598(2.0)$ & \\
\hline Ever, not currently & $20682(60.8)$ & $2562(58.6)$ & $18120(61.1)$ & \\
\hline Currently & $12613(37.1)$ & $1665(38.1)$ & $10947(36.9)$ & \\
\hline $\begin{array}{l}\text { Received all other vaccines to national } \\
\text { schedule } \dagger\end{array}$ & & & & $<0.0001$ \\
\hline No & $20333(60.1)$ & $4365(98.3)$ & $15967(54.3)$ & \\
\hline Yes & 13501 (39.9) & $75(1.7)$ & $13425(45.7)$ & \\
\hline Received vitamin A† & & & & $<0.0001$ \\
\hline No & 11655 (34.3) & $2897(67.1)$ & $8758(29.6)$ & \\
\hline Yes & $22298(65.7)$ & $1422(32.9)$ & $20876(70.4)$ & \\
\hline Slept under bed net last night† & & & & $<0.0001$ \\
\hline No & $14329(43.5)$ & $2232(53.2)$ & $12097(42.1)$ & \\
\hline Yes & $18601(56.5)$ & $1964(46.8)$ & $16637(57.9)$ & \\
\hline \multicolumn{5}{|l|}{ Maternal level } \\
\hline Age of mother in years, mean (SD) & $29.00(7.20)$ & $28.98(7.44)$ & $29.00(7.16)$ & 0.88 \\
\hline BMI of mother, $†$ median (IQR) & $21.64(4.06)$ & $21.21(3.35)$ & $21.72(4.16)$ & $<0.0001$ \\
\hline $\begin{array}{l}\text { Number of births in the last } 5 \text { years, median } \\
\text { (IQR) }\end{array}$ & $2(1)$ & $2(1)$ & $1(1)$ & $<0.0001$ \\
\hline Level of education $\dagger$ & & & & $<0.0001$ \\
\hline
\end{tabular}


Table 1 Continued

\begin{tabular}{|c|c|c|c|c|}
\hline Item & $\begin{array}{l}\text { All children } \\
\mathrm{N}=34206\end{array}$ & $\begin{array}{l}\text { Not } B C G \text { vaccinated } \\
n=4441\end{array}$ & $\begin{array}{l}\text { BCG vaccinated } \\
n=29765\end{array}$ & P value $^{*}$ \\
\hline No education, preschool & $12998(38.0)$ & 2432 (54.8) & $10567(35.5)$ & \\
\hline Primary & $13491(39.5)$ & $1587(35.8)$ & $11904(40.0)$ & \\
\hline Secondary or higher & 7692 (22.5) & $418(9.4)$ & $7274(24.5)$ & \\
\hline Mother has partner† & & & & $<0.0001$ \\
\hline No & $9469(27.7)$ & $793(17.9)$ & $8676(29.2)$ & \\
\hline Yes & 24685 (72.3) & $3640(82.1)$ & $21045(70.8)$ & \\
\hline \multicolumn{5}{|l|}{ Household level } \\
\hline Household size, median (IQR) & $6(4)$ & $7(4)$ & $6(4)$ & $<0.0001$ \\
\hline Drinking water quality† & & & & $<0.0001$ \\
\hline Low & $14349(42.0)$ & $2739(61.7)$ & $11610(39.0)$ & \\
\hline Medium & 10954 (32.0) & $1052(23.7)$ & 9902 (33.3) & \\
\hline High & $8897(26.0)$ & $649(14.6)$ & $8248(27.7)$ & \\
\hline Toilet facility quality $\dagger$ & & & & $<0.0001$ \\
\hline Low & 22635 (66.2) & $3330(75.1)$ & 19305 (64.9) & \\
\hline Medium & $9619(28.1)$ & $911(20.5)$ & 8709 (29.3) & \\
\hline High & $1942(5.7)$ & $195(4.4)$ & $1747(5.9)$ & \\
\hline IWI, median (IQR) & $23.26(24.95)$ & $17.18(16.28)$ & $24.12(26.08)$ & $<0.0001$ \\
\hline Urbanisation level & & & & $<0.0001$ \\
\hline Urban & 9313 (27.2) & $600(13.5)$ & 8713 (29.3) & \\
\hline Rural & $24893(72.8)$ & 3840 (86.5) & 21052 (70.7) & \\
\hline
\end{tabular}

Data are displayed as $\mathrm{n}(\%)$ unless otherwise indicated.

*Students' t-test for normally distributed continuous variables, Somers' $D$ test for non-normally distributed continuous variables, and $\chi^{2}$ test for categorical variables.

†Based on different $\mathrm{n}$ due to missings in the control variables, which were accounted for by the dummy variable adjustment procedure.

BCG, bacillus Calmette-Guérin; IWI, International Wealth Index; RDT, rapid diagnostic test.

discordant RDT-positive, thick-smear negative group $(14.1 \%$ (3674/26131)); however, since the main explanation for this combination would be a treated malaria infection, we did not alter their malaria status. Findings were, furthermore, robust to the exclusion of children 6 months of age or younger who are likely protected by maternal antimalarial antibodies $\left(\mathrm{aOR}_{\mathrm{card}}=0.87,95 \%\right.$ CI 0.81 to $0.92 ; \mathrm{aOR}_{\text {recall }}=0.96,95 \%$ CI 0.91 to 1.01$)$. Restricting the analysis to regions with suboptimal BCG coverage $(\leq 90 \%)$ slightly enhanced the association of BCG both among all children $\left(\mathrm{aOR}_{\text {card }}=0.82,95 \%\right.$ CI 0.74 to $0.92 ; \mathrm{aOR}_{\text {recall }}=0.93,95 \%$ CI 0.89 to 0.96 ) and those over 6 months of age $\left(\mathrm{aOR}_{\text {card }}=0.81,95 \%\right.$ CI 0.73 to 0.89 ; $\mathrm{aOR}_{\text {recall }}=0.92,95 \% \mathrm{CI} 0.89$ to 0.95 ) (figure $2 \mathrm{~B}$ and online supplementary figure 1 ).

\section{Timing of vaccination}

There was no linear time-dependent association between BCG and malaria (aOR=1.00, 95\% CI 0.98 to 1.02) (figure 2C). Inclusion of non-linear time dependent effects, with a categorical timing of BCG variable, did show a small trend for time dependency (figure 2D). The smallest association was seen for vaccination at date of birth $(\mathrm{aOR}=0.93,95 \%$ CI 0.82 to 1.04$)$ and the largest for vaccination was seen more than 1 month after birth (aOR $=0.86,95 \%$ CI 0.78 to 0.96$)$, although differences between the time categories were non-significant.

Although timing of vaccination as such did not influence the association, we did find an interaction with the age of the child. We observed that the association became slightly stronger with each month of age $\left(\mathrm{aOR}_{\text {card }}=0.996\right.$, $95 \%$ CI 0.993 to 0.999 ) (figure $2 \mathrm{E}$ ). The association did not differ by sex of the child $\left(\mathrm{aOR}_{\text {card }}=0.99,95 \%\right.$ CI 0.89 to 1.10$)$.

\section{Symptomatic and asymptomatic malaria}

Of all malaria-positive children, 31.7\% (3890/12 285) was reported to have concomitant fever and were classified as having symptomatic malaria. The other 8395 children did not have concomitant fever and were thus classified as having asymptomatic malaria. The associations with BCG were similar for both symptomatic $\left(\mathrm{aOR}_{\text {card }}=0.89,95 \%\right.$ CI 0.78 to 1.01$)$ and asymptomatic $\left(\mathrm{aOR}_{\text {card }}=0.86,95 \%\right.$ CI 0.81 to 0.92 ) (figure $2 \mathrm{~F}$ ). BCG vaccination was not differently associated with symptomatic or asymptomatic malaria $\left(\mathrm{aOR}_{\text {card }}=1.12,95 \%\right.$ CI 0.96 to 1.30 ). 

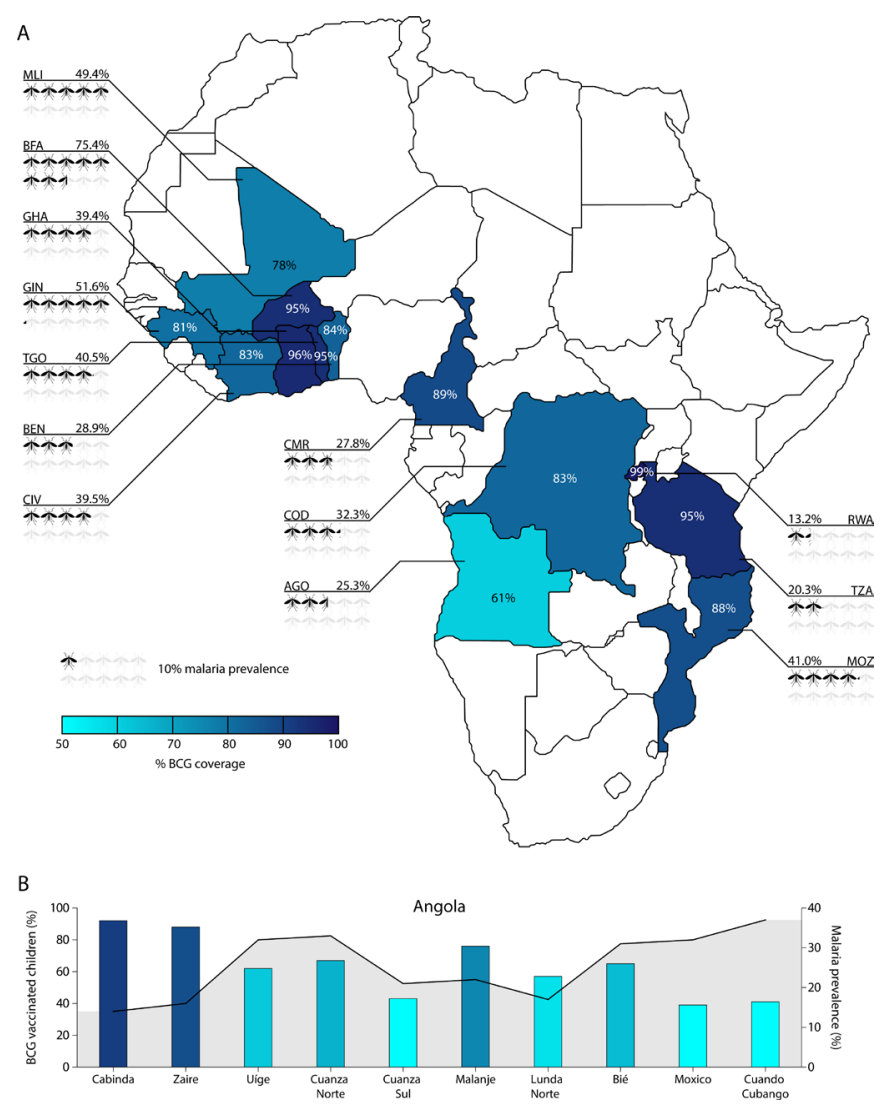

Figure 1 BCG coverage and malaria prevalence distribution between and within 13 sub-Saharan African countries. (A) Distribution of both BCG vaccination coverage and malaria prevalence per country are depicted. (B) Overlay chart of BCG coverage (bars) and malaria prevalence (line) per included region of Angola. BCG, bacillus Calmette-Guérin. ISO codes: ago, Angola; BEN, Benin; BFA, Burkina Faso; CIV, Ivory Coast; CMR, Cameroon; cod, Democratic Republic of the Congo; GHA, Ghana; Gin, guinea; MLI, Mali; MOZ, Mozambique; RWA, Rwanda; TZA, United Republic of Tanzania; TGO, Togo.

\section{DISCUSSION}

In this large DHS survey study among children under the age of 5 years in SSA, we found evidence that BCG vaccination could protect against malaria infection. BCG reduced the odds on malaria independent of timing of vaccination. This effect seemed prolonged, as the association became more beneficial with increasing child's age. The fact that associations were similar regardless of symptomatic status suggests that good BCG vaccination practice might also contribute to elimination of the disease.

\section{Strengths and limitations}

A considerable advantage of this study is the inclusion of 34206 children from 13 different countries in West, East and Central Africa with varying BCG coverage and malaria prevalence. This provided a considerable and representative population of SSA, making it more likely that results will be generalisable to children under the age of five living in other SSA countries. Furthermore, the association between BCG and malaria remained after
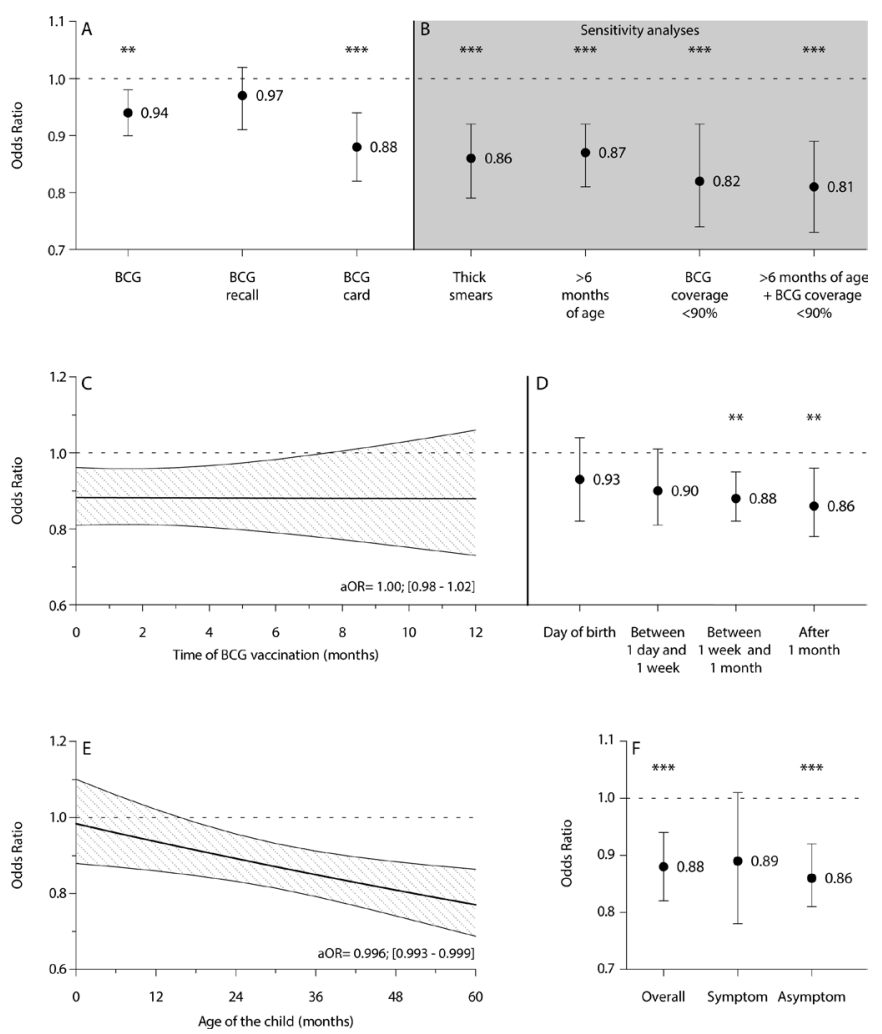

Figure 2 Associations between BCG vaccination and malaria prevalence. (A) aOR of BCG vaccination on malaria prevalence, overall and subdivided by the source of BCG data (NO vaccination as reference). (B) Sensitivity analyses of BCG vaccination data derived from card with thicksmear microscopy as diagnostic, removal of children likely protected by maternal antimalarial antibodies (age $\leq 6$ months), removal of areas with a BCG coverage of $\geq 90 \%$ and a combination of the latter two. (C,D) Time dependency of the association between BCG vaccination and malaria prevalence with a continuous variable (C) and a categorical variable (D) of timing. (E) Association between $B C G$ vaccination derived from card and malaria prevalence related to the age of the child (interaction term age of child in months $\times$ BCG vaccination). (F) Association between BCG vaccination derived from card and malaria prevalence, overall and separate for symptomatic and asymptomatic malaria (symptomatic malaria was based on positive rapid diagnostic test and maternal recall of fever in the past 2 weeks). All results are depicted as $\mathrm{aORs} \pm 95 \% \mathrm{Cls}$ retrieved from multilevel logistic regression analyses with four levels (child, cluster, region and country) with correction for age, birth order, sex, twin, place of delivery, size at birth, preceding birth interval, weight-for-age $z$-score, breastfeeding, other vaccinations, vitamin $A$ supplementation, use of bed nets, maternal age, maternal body mass index, number of births by the mother in the last 5 years, maternal level of education, maternal partner, household size, quality of drinking water, quality of toilet facility, international wealth index and urbanisation level. ${ }^{* \star} \mathrm{P}<0.01,{ }^{* \star *} \mathrm{P}<0.001$. aOR, adjusted OR; BCG, bacillus Calmette-Guérin.

controlling for numerous possible confounders on the level of the child, mother and household, and was robust to removal of regions with BCG coverage of $>90 \%$ or 
Table 2 Full model of the association between BCG and malaria with crude and aORs

\begin{tabular}{lllllll}
\hline Item & Coefficient & SE & Crude OR & P value & aOR & \\
\hline Child level & & & & & &
\end{tabular}

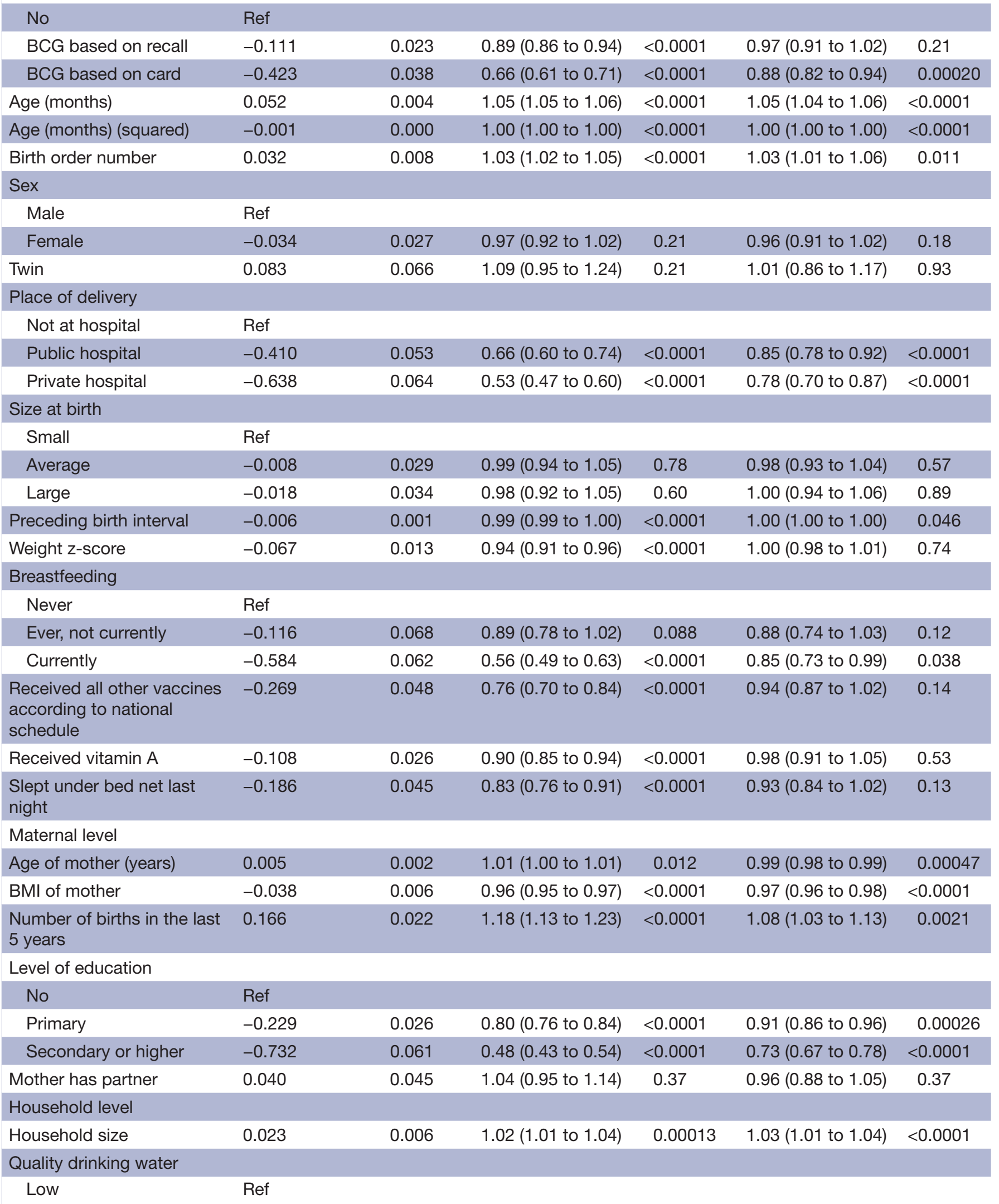




\begin{tabular}{|c|c|c|c|c|c|c|}
\hline Item & Coefficient & SE & Crude OR & $P$ value & aOR & $P$ value \\
\hline Medium & -0.213 & 0.047 & 0.81 (0.74 to 0.89 ) & $<0.0001$ & 1.02 (0.94 to 1.11$)$ & 0.65 \\
\hline High & -0.302 & 0.056 & 0.74 (0.66 to 0.83$)$ & $<0.0001$ & 1.02 (0.90 to 1.15$)$ & 0.76 \\
\hline \multicolumn{7}{|c|}{ Quality toilet facility } \\
\hline Low & Ref & & & & & \\
\hline Medium & -0.486 & 0.057 & 0.62 (0.55 to 0.69$)$ & $<0.0001$ & 0.85 (0.78 to 0.92$)$ & $<0.0001$ \\
\hline High & -0.964 & 0.161 & 0.38 (0.28 to 0.52$)$ & $<0.0001$ & 0.88 (0.77 to 1.01$)$ & 0.060 \\
\hline IWI & -0.004 & 0.003 & 1.00 (0.99 to 1.00$)$ & 0.18 & 1.00 (0.99 to 1.00$)$ & 0.50 \\
\hline IWI squared & $-0.000^{*}$ & 0.000 & 1.00 (1.00 to 1.00$)$ & $<0.0001$ & 1.00 (1.00 to 1.00$)$ & $<0.0001$ \\
\hline \multicolumn{7}{|c|}{ Urbanisation level } \\
\hline Urban & Ref & & & & & \\
\hline Rural & 1.179 & 0.102 & 3.25 (2.66 to 3.97 ) & $<0.0001$ & 1.91 (1.63 to 2.25$)$ & $<0.0001$ \\
\hline
\end{tabular}

${ }^{*}$ Negative sign indicates a significant negative coefficient. Model is a multilevel model including four levels (child, cluster, region and country). aOR, adjusted OR; BCG, Bacillus Calmette-Guérin; IWI, International Wealth Index.

children likely protected by maternal antimalarial antibodies and to the inclusion of thick-smear malaria diagnosis.

Limitations of this study are inherent to the observational character of DHS surveys. Even after we controlled for confounding on several levels in all analyses, residual unmeasured confounders cannot be ruled out. One major confounder we lacked information on is the HIV status of the children. ${ }^{2324}$ However, most of the included countries do not have the means to detect HIV status at birth and WHO recommends administering BCG vaccination to all infants under those circumstances. ${ }^{24}$ Combined with the fact that over one-third of births occurred outside of the hospital, it seems unlikely that children would have been withheld BCG vaccination because of their HIV status.

Other limitations of surveys are their susceptibility to recall bias and incompleteness of data. For BCG vaccination, recall bias was resolved by stratifying the analysis by source of information. Missing data were limited for individual covariates, but a substantial number of children had missing values when all covariates were combined (online supplementary table 4 ). Nevertheless, results remained similar after we restricted the analysis to children without missing values on any of the covariates (online supplementary table 5).

\section{Non-specific BCG effects on malaria}

Many studies have already been performed on the NSEs of BCG in relation to mortality and morbidity, ${ }^{6-12}$ but its relationship with malaria infection is less clear. Despite early evidence of a protective effect in mice, ${ }^{13}$ studies reporting the effects of BCG on malaria in humans are scarce. Two studies in Guinea-Bissau addressed the effect of BCG on malaria, with varying results. Rodrigues $e t \mathrm{al}^{16}$ found no effect of BCG revaccination on malaria incidence, while Roth $e t a l^{15}$ found a reduction in malaria mortality for children with a BCG scar. Since we neither looked at revaccination nor examined malaria mortality, it is hard to draw parallels to these findings. In a more comparable study, Hollm-Delgado et al contradicted our findings, as they concluded that BCG increased the odds on malaria. ${ }^{17}$ However, BCG coverage in their study was over $99 \%$, with only 41 BCG unvaccinated children, thus making any statements on a relation between BCG and malaria inconclusive. Recently, we have provided support for a protective effect of BCG vaccination on malaria in a controlled human malaria infection model. In approximately half of the individuals vaccinated with BCG, there was a strong activation of innate immune mechanisms, which was associated with decreased parasitaemia. ${ }^{14}$

\section{Timing of vaccination}

The non-significant effect of timing of BCG was relatively unexpected, since we have previously found associations between timing of BCG vaccination and linear growth. ${ }^{12}$ This difference was not attributable to the distribution of timing, as analysis of stunting in the current study population confirmed this time dependency, with later administration increasing the odds of stunting $(\mathrm{aOR}=1.04$, $95 \%$ CI 1.02 to 1.06 ) (online supplementary figure 2), ensuring that the absence of time dependency for malaria was not attributable to the dataset used in the current study. One possibility for the discrepancy with malaria could be due to excess inflammation induced by late vaccination and its relative significance to the outcome parameter. ${ }^{12}$ Linear growth is sensitive to excess inflammation as it suppresses the expression of growth hormone/insulin-like growth factor- $1 .{ }^{25}$ Parasite suppression or clearance would be, however, more influenced by immune tolerance than by excess inflammation. ${ }^{26}$

Nevertheless, early vaccination would still maximise a protective effect. Even though timing of vaccination as such did not influence the association with malaria, we found that the association became more beneficial with age. An explanation for this could be a positive feedback loop between BCG facilitated parasite clearance, through 


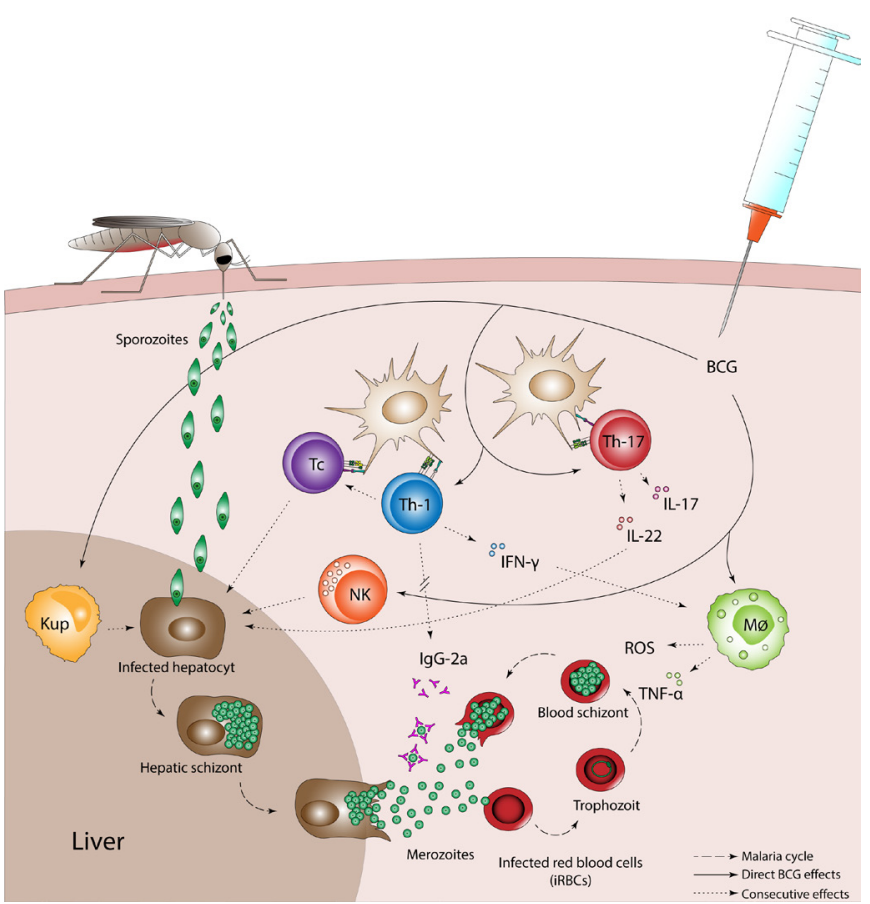

Figure 3 Possible targets via which BCG could influence malaria infection. BCG vaccination induces increased innate immune activation and a Th1/Th 17 cell biassed immune response which could affect the malaria parasite in both liver and blood stages. Stimulation of Tc and NK cells can clean up infected hepatocytes. Furthermore, production of IL-22 by Th17 cells increases innate immune responses of hepatocytes, and BCG-trained Kupffer cells can reduce sporozoite development. Direct and Th1/Th17 promoted activation of monocytes also leads to increased production of proinflammatory cytokines IFN- $\gamma$ and TNF- $\alpha$ and has been linked to the production of IgG-2a antibodies for neutralisation of merozoites. At their turn, proinflammatory cytokines induce killing of infected red blood cells by promoting macrophages to produce reactive oxygen species. BCG, bacillus Calmette-Guérin; IFN- $\gamma$, interferon gamma; IL, interleukin; Kup, Kupffer cell; M $\varphi$, macrophage; NK, natural killer cell; Tc, cytotoxic T-cell; th, T-helper cell; TNF- $\alpha$, tumour necrosis factor alpha.

mechanisms described further, and an accompanying increased memory production, ${ }^{27}$ enhancing the effect with every parasite encounter.

\section{Mechanistic pathways mediating the effects of BCG vaccination}

One underlying mechanism of how BCG reduces malaria prevalence is complex because of the many modulating effects BCG has on the immune system, as well as the different stages of the malaria parasite. Therefore, we propose several targets by which BCG could reduce malaria infection (figure 3). It should be mentioned that these mechanisms are only proposed targets and that the effects of BCG on the immune system in relation to malaria infection are far from understood.

BCG vaccination of healthy adults increased responsiveness of innate immune cells, a process termed 'trained immunity' and biassed the $\mathrm{T}$ helper (Th) response towards Th1/Th17. ${ }^{28-30}$ In the liver stage, the Th1/Th17stimulated production and activation of cytotoxic $\mathrm{T}$ cells, together with BCG-enhanced natural killer (NK) cell activity, can clean up infected hepatocytes. In a controlled human malaria infection, we indeed demonstrated that in half of the individuals vaccinated with BCG, NK and $\mathrm{T}$ cell activities were increased and associated with lower parasitaemia. ${ }^{14}$ In addition, BCG-trained Kuppfer cells and interleukin-22-induced innate immune responses of hepatocytes could reduce sporozoite development. ${ }^{31} 32$ In the blood stage, Th1 responses have been linked to neutralisation of merozoites by IgG-2a antibodies, ${ }^{33}$ and activation of macrophages leads to increased production of proinflammatory cytokines and enhanced killing of infected red blood cells. ${ }^{28} 293334$

\section{Implications}

Although definitive causal relations cannot be derived from our data, the robustness of the findings in this large and representative population strengthens our belief in this newly found association. If the association between BCG and malaria would be attributable to the NSEs of $\mathrm{BCG}$, the average reduction of malaria prevalence in our data would be a modest $1.2 \%$. However, regions with low BCG coverage would have reductions of up to $9.6 \%$ (online supplementary table 6 with accompanying text). This is important as many of the countries suffering most from the global malaria burden have suboptimal BCG coverages at best. ${ }^{2}$

Furthermore, the similar association for asymptomatic malaria highlights a possible role for BCG in areas moving towards malaria elimination, since the relative contribution of asymptomatic malaria to the infectious reservoir in these areas is high. ${ }^{3}$ Although our finding has several limitations (eg, subjective nature of symptomatic diagnosis, missing of submicroscopic infections and missing gametocyte measurement), its possible importance warrants further investigation.

These implications are substantial in the perspective of the stalled reduction of global malaria burden and pressing movements towards malaria elimination. ${ }^{2}$ If confirmed in further research, improvement in global malaria burden may be achieved through this established and cheap vaccine with relatively small and achievable efforts.

\section{Author affiliations}

${ }^{1}$ Department of Internal Medicine, Radboud Center for Infectious Diseases, Radboud University Medical Center, Nijmegen, The Netherlands

${ }^{2}$ Odense Patient data Explorative Network (OPEN), Institute of Clinical Research, University of Southern Denmark and Odense University Hospital, Odense, Denmark ${ }^{3}$ Bandim Health Project, Research Center for Vitamins and Vaccines (CVIVA), Copenhagen, Denmark

${ }^{4}$ Bandim Health Project, INDEPTH Network, Bissau, Guinea-Bissau

${ }^{5}$ Global Data Lab, Institute for Management Research, Radboud University Nijmegen, Nijmegen, The Netherlands

${ }^{6}$ Department for Genomics \& Immunoregulation, Life and Medical Sciences Institute (LIMES), University of Bonn, Bonn, Germany

${ }^{7}$ Human Genomics Laboratory, Craiova University of Medicine and Pharmacy, Craiova, Romania 
Acknowledgements The authors thank the Demographic and Health Surveys program (www.dhsprogram.org) for generously providing the data for this study.

Contributors JS, MN and AvdV conceptualised the study. MB and SvG prepared the database and carried out the first analyses. MB and JS refined and performed the final analyses. QdM, PA, CSB, MN and AvdV contributed to interpretation of the data. MB and SvG wrote the first draft. QdM, JS, PA, CSB, MN and AvdV revised the article critically on intellectual content. All authors contributed to and approved the final manuscript and agreed to be accountable for all aspects of the work in ensuring that questions related to the accuracy or integrity of any part of the work are appropriately investigated and resolved. The corresponding author attests that all listed authors meet authorship criteria and that no others meeting the criteria have been omitted.

Funding CSB was supported by the Danish National Research Foundation (DNRF108) through general support for the CVIVA institute. AvdV was supported by an unrestricted grant from Sysmex Corporation. The funding sources had no role in study design, data collection and analysis, interpretation of data, preparation of the manuscript or decision to submit for publication. The corresponding author had full access to all the data in the study and had final responsibility for the decision to submit for publication.

Map disclaimer The depiction of boundaries on the map(s) in this article do not imply the expression of any opinion whatsoever on the part of BMJ (or any member of its group) concerning the legal status of any country, territory, jurisdiction or area or of its authorities. The map(s) are provided without any warranty of any kind, either express or implied.

Competing interests All authors have completed the Unified Competing Interest form (www.icmje.org/coi_disclosure.pdf) and declare no support from companies for the submitted work; no financial relationships with companies that might have an interest in the submitted work in the previous 3 years; their spouses, partners or children have no financial relationships that may be relevant to the submitted work; no non-financial interests that may be relevant to the submitted work.

Patient consent for publication Not required.

Ethics approval The team executing the Demographic Health Survey ensures protection of human subjects in agreement with local and international laws.

Provenance and peer review Not commissioned; externally peer reviewed.

Data availability statement The data were obtained from the DHS program ( www.dhsprogram.com) under the condition that the authors do not pass the data to other researchers. However, other researchers can apply directly to the DHS program to obtain the data.

Open access This is an open access article distributed in accordance with the Creative Commons Attribution Non Commercial (CC BY-NC 4.0) license, which permits others to distribute, remix, adapt, build upon this work non-commercially, and license their derivative works on different terms, provided the original work is properly cited, appropriate credit is given, any changes made indicated, and the use is non-commercial. See: http://creativecommons.org/licenses/by-nc/4.0/.

ORCID iD

Mike LT Berendsen http://orcid.org/0000-0001-9516-6190

\section{REFERENCES}

1 United Nations (UN). The millennium development goals report 2015. Available: http://www.un.org/millenniumgoals/2015_MDG_Report/ pdf/MDG\%202015\%20rev\%20(July\%201).pdf [Accessed 15 Dec 2017].

2 World Health Organization (WHO). World malaria report 2018. Available: http://apps.who.int/iris/bitstream/handle/10665/275867/ 9789241565653-eng.pdf?ua=1 [Accessed 15 Jan 2019].

3 Tadesse FG, Slater HC, Chali W, et al. The relative contribution of symptomatic and asymptomatic Plasmodium vivax and Plasmodium falciparum infections to the infectious reservoir in a low-endemic setting in Ethiopia. Clin Infect Dis 2018;66:1883-91.

4 Hoffman SL, Vekemans J, Richie TL, et al. The March toward malaria vaccines. Vaccine 2015;33 Suppl 4:D13-23.

5 RTS,S Clinical Trials Partnership. Efficacy and safety of RTS,S/ AS01 malaria vaccine with or without a booster dose in infants and children in Africa: final results of a phase 3 , individually randomised, controlled trial. The Lancet 2015;386:31-45.

6 Aaby P, Martins CL, Garly M-L, et al. Non-Specific effects of standard measles vaccine at 4.5 and 9 months of age on childhood mortality: randomised controlled trial. BMJ 2010;341:c6495.
7 Aaby P, Roth A, Ravn H, et al. Randomized trial of BCG vaccination at birth to low-birth-weight children: beneficial nonspecific effects in the neonatal period? J Infect Dis 2011;204:245-52.

8 Lund N, Andersen A, Hansen ASK, et al. The effect of oral polio vaccine at birth on infant mortality: a randomized trial. Clin Infect Dis 2015;61:1504-11.

9 Aaby P, Andersen A, Martins CL, et al. Does oral polio vaccine have non-specific effects on all-cause mortality? natural experiments within a randomised controlled trial of early measles vaccine. BMJ Open 2016;6:e013335.

10 Higgins JPT, Soares-Weiser K, López-López JA, et al. Association of BCG, DTP, and measles containing vaccines with childhood mortality: systematic review. BMJ 2016;355.

11 Hollm-Delgado M-G, Stuart EA, Black RE. Acute Lower Respiratory Infection Among Bacille Calmette-Guérin (BCG)-Vaccinated Children. Pediatrics 2014;133:e73-81.

12 Berendsen MLT, Smits J, Netea MG, et al. Non-Specific effects of vaccines and stunting: timing may be essential. EBioMedicine 2016;8:341-8.

13 Clark IA, Allison AC, Cox FE. Protection of mice against Babesia and Plasmodium with BCG. Nature 1976;259:309-11.

14 Walk J, de Bree LCJ, Graumans W, et al. Outcomes of controlled human malaria infection after BCG vaccination. Nat Commun 2019;10:874.

15 Roth A, Gustafson P, Nhaga A, et al. Bcg vaccination scar associated with better childhood survival in Guinea-Bissau. Int $J$ Epidemiol 2005;34:540-7.

16 Rodrigues A, Schellenberg JA, Roth A, et al. Revaccination with Bacillus Calmette-Guerin (BCG) vaccine does not reduce morbidity from malaria in African children. Trop Med Int Health 2007:12:224-9.

17 Hollm-Delgado M-G, Piel FB, Weiss DJ, et al. Vitamin A supplements, routine immunization, and the subsequent risk of Plasmodium infection among children under 5 years in sub-Saharan Africa. Elife 2015;4:e03925.

18 Demographic Health Survey (DHS) Program. Who we are. Available: https://dhsprogram.com/Who-We-Are/About-Us.cfm [Accessed 07 Mar 2018].

19 Smits J, Steendijk R. The International wealth index (IWI). Soc Indic Res 2015;122:65-85.

20 Allison PD. Missing data. London: Sage Publications Ltd, 2001

21 United Nations, Department of Economic and Social Affairs (UNDESA). World population prospects 2017. Available: https:// population.un.org/wpp/, custom data acquired via website [Accessed 12 Oct 2018].

22 Abba K, Deeks JJ, Olliaro PL, et al. Rapid diagnostic tests for diagnosing uncomplicated $P$. falciparum malaria in endemic countries. Cochrane Database Syst Rev 2011;64.

23 Cohen C, Karstaedt A, Frean J, et al. Increased prevalence of severe malaria in HIV-infected adults in South Africa. Clin Infect Dis 2005;41:1631-7.

24 World Health Organization (WHO). Weekly epidemiological record. WER 2007:82:17-24

25 Prendergast AJ, Rukobo S, Chasekwa B, et al. Stunting is characterized by chronic inflammation in Zimbabwean infants. PLOS One 2014;9:e86928.

26 Kurup SP, Obeng-Adjei N, Anthony SM, et al. Regulatory T cells impede acute and long-term immunity to blood-stage malaria through CTLA-4. Nat Med 2017;23:1220-5.

27 Harari A, Vallelian F, Pantaleo G. Phenotypic heterogeneity of antigen-specific CD4 T cells under different conditions of antigen persistence and antigen load. Eur J Immunol 2004;34:3525-33.

28 Kleinnijenhuis J, Quintin J, Preijers F, et al. Bacille Calmette-Guerin induces Nod2-dependent nonspecific protection from reinfection via epigenetic reprogramming of monocytes. Proc Natl Acad Sci U S A 2012;109:17537-42.

29 Kleinnijenhuis J, Quintin J, Preijers F, et al. Long-Lasting effects of BCG vaccination on both heterologous Th1/Th17 responses and innate trained immunity. J Innate Immun 2014:6:152-8.

30 Kleinnijenhuis J, Quintin J, Preijers F, et al. Bcg-Induced trained immunity in NK cells: role for non-specific protection to infection. Clin Immunol 2014;155:213-9.

31 Wen-yue X, Xing-xiang W, Jie Q, et al. Plasmodium yoelii: influence of immune modulators on the development of the liver stage. Exp Parasitol 2010;126:254-8.

32 Wolk K, Kunz S, Witte E, et al. II-22 increases the innate immunity of tissues. Immunity 2004;21:241-54.

33 Matsumoto $\mathrm{S}$, Yukitake $\mathrm{H}$, Kanbara $\mathrm{H}$, et al. Mycobacterium bovis Bacillus Calmette-Guérin induces protective immunity against infection by Plasmodium yoelii at blood-stage depending on shifting immunity toward Th1 type and inducing protective IgG2a after the parasite infection. Vaccine 2000;19:779-87. 
34 Ockenhouse CF, Shear HL. Oxidative killing of the intraerythrocytic

Immunol 1984;132:424-31.

malaria parasite Plasmodium yoelii by activated macrophages. $J$ 